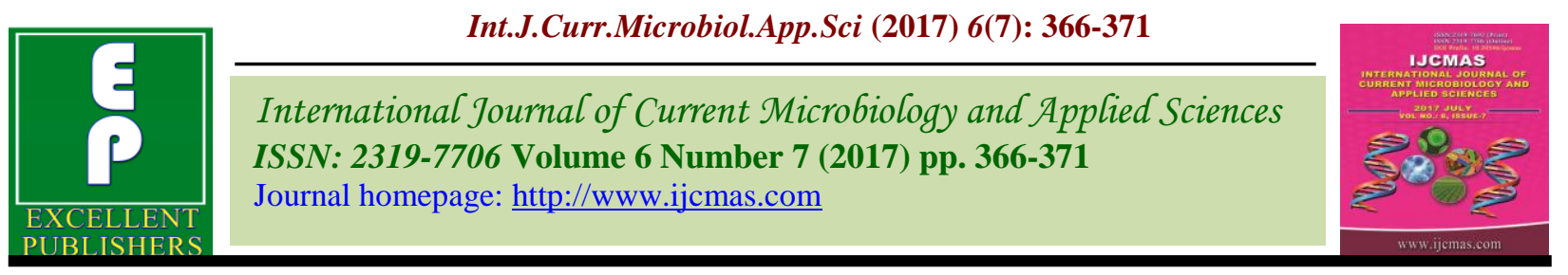

Original Research Article

https://doi.org/10.20546/ijcmas.2017.607.043

\title{
Comparative Biology of Spodoptera litura on Vegetable and Grain Soybean [Glycine max (L.) Merrill]
}

\author{
C. Manja Naik, K. Nataraj* and G.T. Santhoshakumara \\ AICRP on Soybean, ZARS, GKVK, University of Agricultural Sciences, \\ Bangalore 560 065, Karnataka, India \\ *Corresponding author
}

\begin{tabular}{|c|c|}
\hline & A B S T R A C T \\
\hline & \multirow{5}{*}{$\begin{array}{l}\text { The studies on the comparative biology of } S \text {. litura were carried out in laboratory } \\
\text { on vegetable and grain soybean. The duration of egg, larva, prepupa and pupal } \\
\text { period lasted for } 3.13,16.36,1.30 \text { and } 8.48 \text { days respectively on vegetable } \\
\text { soybean. Where has different stages viz., the egg, larva, pre pupa and pupal period } \\
\text { was lasted in } 2.04,14.39,0.91 \text { and } 7.95 \text { days respectively o grain soybean. The } \\
\text { fecundity was } 279.50 \text { in on vegetable soybean. The mean longevity of adult with } \\
\text { food ( } 10 \% \text { honey solution) was ranged between } 9.16 \text { to } 9.16 \text { to } 9.23 \text { and } 5.20 \text { to } \\
5.40 \text { days in female and male moth. The mean longevity of female and male moth } \\
\text { without food was ranged between } 5.10 \text { to } 5.50 \text { and } 3.90 \text { to } 3.98 \text { days respectively } \\
\text { on vegetable soybean during June to November } 2012 \text {. Mean total life cycle lasted } \\
\text { between } 29.88 \text { and } 32.12 \text { days on vegetable soybean, it was } 27.10 \text { and } 30.14 \text { days } \\
\text { on grain soybean respectively during June to November } 2012 \text {. }\end{array}$} \\
\hline Keywords & \\
\hline $\begin{array}{l}\text { Biology, S. litura, } \\
\text { Vegetable and } \\
\text { grain soybean. }\end{array}$ & \\
\hline Article Info & \\
\hline $\begin{array}{l}\text { Accepted: } \\
\text { 04 June } 2017 \\
\text { Available Online: } \\
\text { 10 Julv } 2017\end{array}$ & \\
\hline
\end{tabular}

\section{Introduction}

India is one of the major oilseeds producing country in the world and these are the second largest agricultural commodity in India after cereals occupying $13-14 \%$ of gross cropped area (Sreekanth et al., 2013). Soybean (Glycine max (L.) Merrill) is an oil seed crop contains about 40-42 per cent protein (Netam et al., 2013).

\section{Spodoptera litura (Fab.) (Lepidoptera:} Noctuidae) is polyphagous pest damaging numerous crops in India and many other countries (Shivayogeshwara et al., 1991). S. litura is also known as tobacco cutworm, or cluster or tobacco caterpillar. It has reached a major status of insect an important pest on soybean [Glycine max (L.) Merrill] and major defoliator vegetable soybean. It infests soybean from mid-August to October in kharif. Incidence of $S$. litura was observed in all the soybean growing parts of northern Karnataka during kharif. During the vegetative stage of the crop it feeds on leaves and on young parts at reproductive stage it feeds on pods (Anonymous, 2007). S. litura was reported to cause 30 to 50 per cent crop loss in Dharwad and Belgaum districts of Karnataka (Patil, 2002). Study of the host plants on the biology of insects is important in understanding host suitability of plant infesting insect species. There have been a number of studies on the biological 
parameters of $S$. litura on different host plants under different environmental conditions, particularly in India (Patel et al., 1986, 1987). In view of several health benefits of soybean, there is growing demand for vegetable soybean in affluent urban population in the country. The vegetable soybean is characteristically rich in sucrose content besides many food grade characters. These characters are bound to influence insect lifecycle, but there is no systematic study on this part. Keeping all these points in view the present investigation on biology of S. litura on vegetable and grain soybean was undertaken.

\section{Materials and Methods}

The biology of the $S$. litura on vegetable and grain soybean was studied under laboratory at room temperature and relative humidity at the Zonal Agricultural Research Station, Gandhi Krishi Vigyana Kendra, University of Agricultural Sciences, Bengaluru during June to November 2012.

\section{Mass rearing of $S$. litura}

The larvae of $S$. litura was collected from the vegetable soybean field and mass reared in the laboratory on vegetable soybean plants. A pair of newly emerged male and female moths obtained from the nucleus culture was introduced in to a wooden cage $(35 \mathrm{x} 25 \mathrm{x} 40$ $\mathrm{cm})$ vegetable soybean plants egg laying. The root portion of the plant was immersed in a container containing water for maintaining the turgidity and freshness of the plant. Cotton swab was dipped in ten per cent honey solution which served as food for the moths Oviposit ion period and fecundity was studied by enclosing ten pairs of newly emerged male and female moths in the wooden rearing cages $(35 \mathrm{~cm} \times 25 \mathrm{~cm} \times 40 \mathrm{~cm})$, with wire mesh on three sides and wooden front door at the rate of one pair per cage for egg laying. The open end of the jar was covered with muslin cloth fastened with rubber band. The egg mass deposited every day was collected till the death of female moth. For studies on incubation period, freshly laid eggs were kept in petri dishes provided with wet blotting paper at the bottom which protect the eggs from desiccation.

The larval instars were noted by transferring freshly hatched neonate larvae into specimen tubes individually. Fresh vegetable soybean/grain soybean leaves were provided as food. The food was changed after every 24 h.

The first instar larvae of $S$. litura were reared on both vegetable and grain soybean host plants. Fifty larvae were used in batches. The food was changed daily. The incubation period, larval period, per cent pupation, fresh pupal weight within $24 \mathrm{~h}$ of pupation, pupal duration, total developmental period, per cent adult emergence and mean number of eggs laid by female were recorded on each host. The experiment was conducted from June to November, 2012 and data was generated for five generations.

\section{Results and Discussion}

The observations on biology of S. litura on vegetable and grain soybean during June to November (2012) under laboratory are presented in (Table 1). The, egg, larva, prepupa and pupal period was lasted for 3.13, 16.34, 1.30 and 8.48 days respectively, on vegetable soybean. Whereas, the egg, larva, pre-pupa and pupal period was completed in $2.84,14.39,0.91$ and 7.95 days respectively, on grain soybean. The present findings are similar to the observations made by Kulkarni (1989) on the biology of S. litura on ground nut. The $S$. litura deposited eggs in batches on tender leaves and covered with the abdominal scales, the larvae moulted for five times and completed six instars. Pupation occurred at six to eight $\mathrm{cm}$ depth in soil. 
Table.1 Biology of S. litura on vegetable soybean and grain soybean under laboratory (June - November 2012)

\begin{tabular}{|c|c|c|c|c|c|c|c|c|c|c|c|}
\hline \multirow[t]{2}{*}{ Month } & \multirow[t]{2}{*}{ Host } & \multicolumn{2}{|c|}{$\begin{array}{c}\text { Incubation } \\
\text { period (days) }\end{array}$} & \multicolumn{2}{|c|}{$\begin{array}{c}\text { Larval period } \\
\text { (days) }\end{array}$} & \multicolumn{2}{|c|}{$\begin{array}{c}\text { Pre-pupal period } \\
\text { (days) }\end{array}$} & \multicolumn{2}{|c|}{$\begin{array}{c}\text { Pupal period } \\
\text { (days) }\end{array}$} & \multicolumn{2}{|c|}{ Total life cycle (days) } \\
\hline & & Range & Mean & Range & Mean & Range & Mean & Range & Mean & Range & Mean \\
\hline \multirow[t]{2}{*}{ June - July } & $\begin{array}{l}\text { Vegetable } \\
\text { soybean }\end{array}$ & $2.45-3.62$ & 3.13 & $13.13-17.44$ & 16.34 & $0.65-1.76$ & 1.30 & $7.36-9.17$ & 8.48 & $26.84-31.67$ & 30.10 \\
\hline & Grain soybean & $2.32-3.50$ & 2.89 & $13.10-16.12$ & 14.39 & $0.54-1.22$ & 0.91 & $6.22-8.12$ & 7.95 & $24.68-30.13$ & 28.13 \\
\hline \multirow[t]{2}{*}{ July - August } & $\begin{array}{l}\begin{array}{l}\text { Vegetable } \\
\text { soybean }\end{array} \\
\end{array}$ & $2.45-3.52$ & 3.12 & $13.20-17.40$ & 16.32 & $0.65-1.74$ & 1.29 & $7.36-9.16$ & 8.46 & $24.66-30.56$ & 29.90 \\
\hline & Grain soybean & $2.20-3.43$ & 2.84 & $13.12-15.14$ & 13.22 & $0.52-1.14$ & 0.88 & $6.20-8.10$ & 7.90 & $23.90-29.13$ & 27.10 \\
\hline \multirow[t]{2}{*}{$\begin{array}{l}\text { August - } \\
\text { September }\end{array}$} & $\begin{array}{l}\begin{array}{l}\text { Vegetable } \\
\text { soybean }\end{array} \\
\end{array}$ & $2.35-3.52$ & 3.11 & $13.20-17.40$ & 16.36 & $0.65-1.74$ & 1.28 & $7.36-9.17$ & 8.44 & $26.66-32.5$ & 32.12 \\
\hline & Grain soybean & $2.23-3.46$ & 2.89 & $12.90-16.22$ & 14.36 & $0.54-1.25$ & 0.91 & $7.32-8.0$ & 7.94 & $24.32-31.10$ & 30.14 \\
\hline \multirow[t]{2}{*}{$\begin{array}{l}\text { September - } \\
\text { October }\end{array}$} & $\begin{array}{l}\text { Vegetable } \\
\text { soybean }\end{array}$ & $2.45-3.60$ & 3.18 & $13.10-17.20$ & 14.20 & $0.65-1.76$ & 1.28 & $7.34-9.14$ & 8.42 & $26.80-32.0$ & 30.12 \\
\hline & Grain soybean & $2.10-3.39$ & 2.86 & $13.00-16.15$ & 13.44 & $0.53-1.21$ & 0.90 & $7.28-8.23$ & 7.92 & $24.54-29.34$ & 28.2 \\
\hline \multirow[t]{2}{*}{$\begin{array}{l}\text { October - } \\
\text { November }\end{array}$} & $\begin{array}{l}\text { Vegetable } \\
\text { soybean }\end{array}$ & $2.45-3.62$ & 3.19 & $13.10-17.20$ & 15.26 & $0.65-1.76$ & 1.30 & $7.34-9.14$ & 8.40 & $26.78-31.60$ & 29.88 \\
\hline & Grain soybean & $2.14-3.40$ & 2.84 & $13.10-16.12$ & 13.32 & $0.54-1.19$ & 0.89 & $7.22-8.10$ & 7.90 & $24.32-29.12$ & 28.12 \\
\hline
\end{tabular}

Table.2 Adult longevity (days) of S. litura on vegetable soybean under laboratory (June - November- 2012)

\begin{tabular}{|l|c|c|c|c|c|c|c|c|}
\hline \multirow{2}{*}{ Period of study } & \multicolumn{2}{|c|}{$\begin{array}{c}\text { Longevity with food } \\
\text { (Male) }\end{array}$} & \multicolumn{2}{c|}{$\begin{array}{c}\text { Longevity without food } \\
\text { (Male) }\end{array}$} & \multicolumn{2}{c|}{$\begin{array}{c}\text { Longevity with food } \\
\text { (Female) }\end{array}$} & $\begin{array}{c}\text { Longevity without food } \\
\text { (Female) }\end{array}$ \\
\cline { 2 - 9 } & Range & Mean & Range & Mean & Range & Mean & Range & Mean \\
\hline June - July & $4.16-6.26$ & 5.37 & $3.10-4.56$ & 3.96 & $9.02-9.56$ & 9.21 & $3.20-5.22$ & 5.10 \\
\hline July - August & $4.12-6.22$ & 5.20 & $3.08-4.52$ & 3.92 & $9.02-9.54$ & 9.20 & $3.22-5.24$ & 5.00 \\
\hline August - September & $4.18-6.28$ & 5.40 & $3.12-4.58$ & 3.98 & $9.05-9.58$ & 9.23 & $3.25-5.25$ & 5.13 \\
\hline September - October & $4.16-6.24$ & 5.22 & $3.10-4.54$ & 3.92 & $9.03-9.52$ & 9.18 & $3.24-5.22$ & 5.50 \\
\hline October - November & $4.14-6.22$ & 4.20 & $3.12-4.54$ & 3.90 & $9.04-9.51$ & 9.16 & $3.22-5.20$ & 5.40 \\
\hline
\end{tabular}


Table.3 Mean comparative of biology of S. litura on vegetable soybean and grain soybean

\begin{tabular}{|c|c|c|c|c|c|c|c|c|c|c|c|c|c|}
\hline Month & Host & $\begin{array}{c}\text { Pre- } \\
\text { oviposition } \\
\text { period } \\
\text { (days) }\end{array}$ & $\begin{array}{c}\text { Ovi- } \\
\text { position } \\
\text { period } \\
\text { (days) }\end{array}$ & $\begin{array}{c}\text { No. of } \\
\text { eggs } \\
\text { per } \\
\text { female }\end{array}$ & $\begin{array}{l}\text { Incu- } \\
\text { bation } \\
\text { period } \\
\text { (days) }\end{array}$ & $\begin{array}{l}\text { Larval } \\
\text { period } \\
\text { (days) }\end{array}$ & $\begin{array}{l}\text { Pre - } \\
\text { pupal } \\
\text { period } \\
\text { (days) }\end{array}$ & $\begin{array}{l}\text { Per cent } \\
\text { pupation }\end{array}$ & \begin{tabular}{|} 
Length \\
of \\
pupa \\
(mm)
\end{tabular} & $\begin{array}{l}\text { Weight } \\
\text { of } \\
\text { pupa } \\
\text { (mg) }\end{array}$ & $\begin{array}{l}\text { Pupal } \\
\text { period } \\
\text { (days) }\end{array}$ & $\begin{array}{c}\text { Total } \\
\text { develop- } \\
\text { mental } \\
\text { period } \\
\text { (days) }\end{array}$ & $\begin{array}{c}\text { Per cent } \\
\text { adult } \\
\text { emergence }\end{array}$ \\
\hline \multirow[t]{2}{*}{$\begin{array}{l}\text { June - } \\
\text { July }\end{array}$} & $\begin{array}{l}\text { Vegetable } \\
\text { soybean }\end{array}$ & 2.06 & 3.59 & 278.50 & 3.13 & 16.34 & 1.30 & 82.20 & 18.05 & 380.40 & 8.48 & 30.10 & 75.06 \\
\hline & $\begin{array}{l}\text { Grain } \\
\text { soybean }\end{array}$ & 2.04 & 3.54 & 191.0 & 2.89 & 14.39 & 0.91 & 80.00 & 17.07 & 372.20 & 7.95 & 28.13 & 72.02 \\
\hline \multirow[t]{2}{*}{$\begin{array}{l}\text { July- } \\
\text { August }\end{array}$} & $\begin{array}{l}\text { Vegetable } \\
\text { soybean }\end{array}$ & 2.08 & 3.56 & 274.0 & 3.12 & 16.32 & 1.29 & 80.00 & 18.04 & 380.00 & 8.46 & 29.90 & 74.02 \\
\hline & $\begin{array}{l}\text { Grain } \\
\text { soybean }\end{array}$ & 2.02 & 3.52 & 188.0 & 2.84 & 13.22 & 0.88 & 78.00 & 17.03 & 370.00 & 7.90 & 27.10 & 71.04 \\
\hline \multirow[t]{2}{*}{$\begin{array}{l}\text { August- } \\
\text { September }\end{array}$} & $\begin{array}{l}\text { Vegetable } \\
\text { soybean }\end{array}$ & 2.08 & 3.58 & 252.0 & 3.11 & 16.36 & 1.28 & 86.30 & 18.05 & 382.20 & 8.44 & 32.12 & 75.00 \\
\hline & $\begin{array}{l}\text { Grain } \\
\text { soybean }\end{array}$ & 2.04 & 3.54 & 185.0 & 2.89 & 14.36 & 0.91 & 84.00 & 17.06 & 373.00 & 7.94 & 30.14 & 70.10 \\
\hline \multirow[t]{2}{*}{$\begin{array}{l}\text { September } \\
\text { - October }\end{array}$} & $\begin{array}{l}\text { Vegetable } \\
\text { soybean }\end{array}$ & 2.06 & 3.56 & 307.0 & 3.18 & 14.20 & 1.28 & 82.60 & 18.02 & 378.00 & 8.42 & 30.12 & 72.12 \\
\hline & $\begin{array}{l}\text { Grain } \\
\text { soybean }\end{array}$ & 2.06 & 3.52 & 182.0 & 2.86 & 13.44 & 0.90 & 80.00 & 17.02 & 363.00 & 7.92 & 28.20 & 68.40 \\
\hline \multirow[t]{2}{*}{$\begin{array}{l}\text { October - } \\
\text { November }\end{array}$} & $\begin{array}{l}\text { Vegetable } \\
\text { soybean }\end{array}$ & 2.06 & 3.56 & 286.0 & 3.19 & 15.26 & 1.30 & 80.00 & 17.98 & 380.00 & 8.40 & 29.88 & 74.22 \\
\hline & $\begin{array}{l}\text { Grain } \\
\text { soybean }\end{array}$ & 2.04 & 3.52 & 190.0 & 2.84 & 13.32 & 0.89 & 78.00 & 17.00 & 372.00 & 7.90 & 28.12 & 68.00 \\
\hline
\end{tabular}


Table.4 Mean Fecundity of $S$. litura on vegetable soybean under laboratory (June - November 2012)

\begin{tabular}{|c|c|c|c|c|c|c|c|c|c|c|}
\hline & \multicolumn{10}{|c|}{ Days after emergence } \\
\cline { 2 - 10 } Sl. No. & $\mathbf{2}^{\text {nd }}$ & $\mathbf{3}^{\text {rd }}$ & $\mathbf{4}^{\text {th }}$ & $\mathbf{5}^{\text {th }}$ & $\mathbf{6}^{\text {th }}$ & $\mathbf{7}^{\text {th }}$ & $\mathbf{8}^{\text {th }}$ & $\mathbf{9}^{\text {th }}$ & $\mathbf{1 0}^{\text {th }}$ & Total \\
\hline 1 & - & 31 & 36 & 40 & 110 & 70 & - & - & - & 287 \\
\hline 2 & - & - & 30 & 60 & 115 & 65 & - & - & - & 270 \\
\hline 3 & - & - & 40 & 68 & 108 & 40 & 20 & - & - & 276 \\
\hline 4 & - & 15 & 45 & 62 & 112 & 38 & - & - & - & 272 \\
\hline 5 & - & 16 & 47 & 55 & 114 & 32 & - & - & - & 264 \\
\hline 6 & - & 18 & 30 & 66 & 106 & 20 & - & - & - & 240 \\
\hline 7 & - & - & 45 & 70 & 120 & 38 & 21 & - & - & 294 \\
\hline 8 & - & 24 & 46 & 68 & 130 & 32 & 20 & -- & - & 320 \\
\hline 9 & - & 22 & 40 & 56 & 128 & 40 & 28 & - & - & 314 \\
\hline 10 & - & 20 & 32 & 58 & 110 & 38 & - & - & - & 258 \\
\hline Total & - & 146 & 391 & 603 & 1153 & 413 & 89 & - & - & 2795 \\
\hline Average & - & 14.6 & 39.1 & 60.3 & 115.3 & 41.3 & 8.9 & - & - & 279.5 \\
\hline
\end{tabular}

The mean longevity of adult with food (10\% honey solution) was between 9.16 and 9.23 and 5.20 to 5.40 days in female and male moth respectively, and mean longevity of female and male moth without food ranged between 5.10 and 5.50 and 3.90 to 3.98 days respectively, on vegetable soybean during June to November (Table 2). The present observations are in comparison with the studies made by Patel et al., (1987). Preoviposition and oviposition period lasted for 2.06 to 2.08 and 3.56 to 3.59 days on vegetable soybean. However, it was 2.02 to 2.06 and 3.52 to 3.54 days on grain soybean during June to November (Table 3). The fecundity ranged from 240 to 320 eggs with an average of 279.50 eggs per female on vegetable soybean (Table 4 ). The mean total life cycle was ranged between 29.88 and 32.12 days during June to November. The similar developmental period was reported by Thippeswamy (1983) and Kulkarni (1989) on groundnut and soybean.

The study indicated that, comparative biology of S. litura on soybean 1 the preferentiality of host by the insect. S. litura preferred vegetable soybean host than grain soybean host. The biotic potentiality of the pest was high on vegetable soybean compared to grain soybean.

\section{References}

Anonymous, 2007, Soybean, www.ikisan.com, pp. 1-7

Kulkarni, K.A, 1989. Bio ecology and management of $S$. litura (F.) (Lepidoptera: Noctuidae) on groundnut (Arachis hypogaea L.) Ph. D. Thesis, University of Agricultural Science, Dharwad, India.

Netam, H. K., Gupta, R. and Soni, S. (2013). Seasonal incidence of insect pests and their bio-control agents on soybean. IOSR J. Agri. and Veter. Sci., 292: 7-11.

Patel I.S., Shah, A.H and Rote, N. B. 1987. Effect of different food plants on development of leaf eating caterpillar, Spodoptera litura (F.). Gujarat Agricultural University Research Journal 12(2): 57-8.

Patel, I.S., Rote N.B. Shah A.H. and Patel, U.G.1986. Biology of cotton leaf worm, Spodoptera litura (Noctuidae: Lepidoptera) on cotton, Gujarat Agric. 
Uni. Res. J., 11(2) p. 67-68.

Patil, R.H.2002. Evaluation of insect pest management components in soybean eco- system. Ph.D. Thesis, University of Agricultural Science, Dharwad, India.

Shivayogeshwara, B., Millikharjunaiah, H and Krishnaprasad, N.K.1991.Integrated management of Spodoptera litura Fabricius (Noctuidae: Lepidoptera) in FCV tobacco crop. Tobacco Research 17(8): 59-61.

Sreekanth, P., Venkattakumar, R., Kumar,
K.V. and Kamala Jayanthi, (2013). Impact of climate on oilseed production in Andhra Pradesh: A case study to understand regional level influences. $J$. Agrometeorol., 15(Special Issue I): 150156.

Thippeswamy C. 1983. Life history of tobacco caterpillar, S. Litura on groundnut Arachis hypogaea. Agricultural Science Digest 5: 47-9.

\section{How to cite this article:}

Manja Naik, C., K. Nataraj and Santhoshakumara, G.T. 2017. Comparative Biology of Spodoptera litura on Vegetable and Grain Soybean [Glycine max (L.) Merrill]. Int.J.Curr.Microbiol.App.Sci. 6(7): 366-371. doi: https://doi.org/10.20546/ijcmas.2017.607.043 